Article

\title{
On Dynamic Extension of a Local Material Symmetry Group for Micropolar Media
}

\author{
Victor A. Eremeyev ${ }^{1,2, *}$ and Violetta Konopińska-Zmysłowska ${ }^{1}[\mathbb{C}$ \\ 1 Faculty of Civil and Environmental Engineering, Gdańsk University of Technology, 80-233 Gdansk, Poland; \\ violetta.konopinska@pg.edu.pl \\ 2 Research Institute for Mechanics, National Research Lobachevsky State University of Nizhny Novgorod, \\ 603950 Nizhny Novgorod, Russia \\ * Correspondence: vicereme@pg.edu.pl; Tel.: +48-58-3471422
}

Received: 27 August 2020; Accepted: 29 September 2020; Published: 3 October 2020

\begin{abstract}
For micropolar media we present a new definition of the local material symmetry group considering invariant properties of the both kinetic energy and strain energy density under changes of a reference placement. Unlike simple (Cauchy) materials, micropolar media can be characterized through two kinematically independent fields, that are translation vector and orthogonal microrotation tensor. In other words, in micropolar continua we have six degrees of freedom (DOF) that are three DOFs for translations and three DOFs for rotations. So the corresponding kinetic energy density nontrivially depends on linear and angular velocity. Here we define the local material symmetry group as a set of ordered triples of tensors which keep both kinetic energy density and strain energy density unchanged during the related change of a reference placement. The triples were obtained using transformation rules of strain measures and microinertia tensors under replacement of a reference placement. From the physical point of view, the local material symmetry group consists of such density-preserving transformations of a reference placement, that cannot be experimentally detected. So the constitutive relations become invariant under such transformations. Knowing a priori a material's symmetry, one can establish a simplified form of constitutive relations. In particular, the number of independent arguments in constitutive relations could be significantly reduced.
\end{abstract}

Keywords: micropolar continuum; local symmetry group; kinetic constitutive equation; Cosserat continuum; anisotropy

\section{Introduction}

The model of micropolar medium was originally introduced by Cosserat brothers [1]. Recently, it founds various applications for modelling of media with complex inner microstructure such as granular [2,3], porous [4,5], composite [6,7] materials, masonries [8,9], bones [10,11], textiles and beam-lattices [12-16], suspensions [17-19], see also [20-24]. The kinematics of the model is based on two descriptors that are translation and rotation fields. Accordingly, in dynamics we have two independent velocity fields of linear $\mathbf{v}$ and angular $\omega$ velocity. For a hyperelastic medium there is a strain energy density $W$ which depends on two strain measures. Constitutive relations for micropolar solids are discussed in many works, see, e.g., [20-25] and references therein. As in the case of simple (Cauchy) materials assumed symmetry properties can result in further simplifications of constitutive relations. To this end a concept of the local material symmetry group may play an important role as in case of nonlinear elasticity [26]. The definition of the material symmetry group for micropolar materials was proposed by Eringen and Kafadar [20] who included a single microinertia tensor as an argument of constitutive relations. The definition was extended by Eremeyev and Pietraszkiewicz [27] considering additional set of arguments and pseudotensorial nature of the 
wryness tensor. Furthermore, in [28] various systems of strain invariants were obtained for several symmetries and the corresponding constitutive equations of micropolar solids, fluids and subfluids were presented. The analysis of symmetry groups $[20,27,28]$ was performed on the base of the strain energy density, i.e., for statics. On the other hand, in micropolar dynamics the form and possible symmetries of a kinetic energy density may also play an important role.

In the case of simple materials, the form of kinetic energy is rather simple and cannot change symmetry properties. Indeed, for a simple material it has a form

$$
K(\mathbf{v})=\frac{1}{2} \rho_{\kappa} \mathbf{v} \cdot \mathbf{v},
$$

where $\rho_{\kappa}$ is a mass density in a reference placement $\kappa, \mathbf{v}$ is a velocity vector, and the dot denotes the inner (scalar) product of two vectors. Here $K$ depends on $\kappa$ only through the mass density. As a symmetry group consists of transformations which do not change $\rho_{\kappa}, K$ is independent of such transformations.

On the other hand, in rigid body dynamics a form of kinetic energy plays a crucial role for analysis of motions [27,29]. As in case of rigid body dynamics, for micropolar media a kinetic energy density has more general form than for simple materials, its symmetry should be included in a symmetry characterization of a micropolar material. In other words, we characterize a material symmetry considering static and dynamic properties of materials. For example, Eringen $[17,18,20,21]$ included the microinertia tensor in the set of primary parameters and proposed additional evolution law for this tensor. The changes in microinertia properties related to phase transitions were also discussed in [30].

The aim of this paper is to consider a general form of kinetic constitutive equation, i.e., a dependence of a kinetic energy density, and include its symmetries into the definition of the material local symmetry group. The paper is organized as follows. First, in Section 2 we present the general form of the strain energy density of a micropolar medium undergoing finite deformations. Section 3 is devoted to the kinetic constitutive relations. The new definition of the local material symmetry group is given in Section 4. Finally, we discuss the further applications of the proposed approach to other generalized media such as strain-gradient elasticity and micromorphic media.

\section{Strain Energy Density}

Let us consider finite deformations of micropolar body $\mathcal{B}$. The finite deformation of $\mathcal{B}$ can be described by an invertible mapping from a reference placement $\kappa(\mathcal{B})$ to the current placement $\chi(\mathcal{B})(t)$. For a material particle $x \in \mathcal{B}$ considered in $\kappa(\mathcal{B})$ we introduce its position vector $\mathbf{x}$ whereas its orientation is described trough a triple of unit orthogonal vectors $\mathbf{g}_{k}$ called directors. In $\chi(\mathcal{B})$ the position and orientation of the same particle $x$ is given by the position vector $\mathbf{y}$ and directors $\mathbf{d}_{k}$, respectively. As a result, a mapping from $\kappa$ to $\chi$ can be described as follows

$$
\mathbf{u}=\mathbf{u}(\mathbf{x}, t) \equiv \mathbf{y}(\mathbf{x}, t)-\mathbf{x}, \quad \mathbf{H}=\mathbf{H}(\mathbf{x}, t) \equiv \mathbf{d}_{k}(\mathbf{x}, t) \otimes \mathbf{g}_{k}(\mathbf{x}), \quad k=1,2,3,
$$

where $\mathbf{u}$ is the translation vector, $\mathbf{H}$ is the microrotation orthogonal tensor, $\mathbf{H}^{-1}=\mathbf{H}^{T}, t$ is time, $\otimes$ stands for the dyadic product, $T$ means the transpose operation applied to a second-order tensor, and Einstein's summation rule is applied. These two kinematically independent fields $\mathbf{u}(\mathbf{x}, t)$ and $\mathbf{H}(\mathbf{x}, t)$ describe translational and rotational DOFs of the micropolar medium called also Cosserat or polar continuum $[20,21,27,28]$.

For a (hyper)elastic continuum there exists a strain energy density $W_{\kappa}$ per unit volume of $\kappa$. $W_{\kappa}$ has the following form [31]

$$
W_{\kappa}=\widehat{W}_{\kappa}(\mathbf{U}, \mathbf{V} ; \mathbf{x}, \mathbf{B}) \text {, }
$$

where the natural Lagrangian relative stretch $\mathbf{U}$ and wryness $\mathbf{V}$ tensors are defined by the relations

$$
\mathbf{U}=\mathbf{H}^{T} \cdot \mathbf{F}-\mathbf{1}, \quad \mathbf{V}=\frac{1}{2}(\mathbf{1} \times \mathbf{1}):\left(\mathbf{H}^{T} \cdot \operatorname{Grad} \mathbf{H}\right) ; \quad \mathbf{F}=\operatorname{Grad} \mathbf{y} .
$$


Here $\mathbf{F}$ is the deformation gradient tensor [26], $\mathbf{1}$ is the 3D unit tensor, Grad is the referential gradient operator, $\times$ and : stand for the cross and double dot products, respectively. In addition, $\mathbf{B}$ is the referential microstructure curvature tensor defined as

$$
\mathbf{B}=\frac{1}{2} \mathbf{g}_{k} \times \operatorname{Grad} \mathbf{g}_{k}
$$

The form (3) satisfies the material frame-indifference principle [26] discussed also in $[23,31]$ for micropolar continua. Note that using index $\kappa$ in (3) we explicitly underline the possible dependence of the strain energy density on the reference placement $\kappa$. The invariance properties of $W_{\kappa}$ under changes of $\kappa$ were studied in $[27,28]$ in more detail, where on the base of the invariance the definition of the local material symmetry group was given and related simplified constitutive relations were presented.

\section{Kinetic Constitutive Equation}

Differentiating (2) with respect to $t$ we get the linear and angular velocity

$$
\mathbf{v}=\dot{\mathbf{u}}, \quad \boldsymbol{\omega}=-\frac{1}{2}(\mathbf{1} \times \mathbf{1}):\left(\dot{\mathbf{H}} \cdot \mathbf{H}^{T}\right), \quad \dot{\mathbf{H}}=\boldsymbol{\omega} \times \mathbf{H} .
$$

Here the overdot stands for the derivative with respect to $t$.

Let us consider a general possible form of the kinetic energy $K_{\kappa}$ of a micropolar medium. As we have linear $\mathbf{v}$ and angular $\omega$ velocity it is natural to assume that $K_{\kappa}$ depends on $\mathbf{v}$ and $\omega$. Using analogy with rigid body dynamics [29] or with six-parameter shell theory [23,32] we restrict ourselves by quadratic forms of $K_{k}$. So we have

$$
K_{\kappa}=\rho_{\kappa} \widehat{K}_{\kappa}(\mathbf{v}, \omega), \quad \widehat{K}_{\kappa}(\mathbf{v}, \omega)=\frac{1}{2} \mathbf{v} \cdot \mathbf{J}_{0} \cdot \mathbf{v}+\mathbf{v} \cdot \mathbf{J}_{1} \cdot \omega+\frac{1}{2} \omega \cdot \mathbf{J}_{2} \cdot \omega,
$$

where $\rho_{\kappa}$ is a mass density in a reference placement $\kappa, \mathbf{J}_{0}, \mathbf{J}_{1}$ and $\mathbf{J}_{2}$ are second-order tensors called microinertia tensors. $\mathbf{J}_{0}$ and $\mathbf{J}_{2}$ are symmetric tensors. From both physical and mathematical points of view $K_{\kappa}$ should be a positive function

$$
\widehat{K}_{\kappa}(\mathbf{v}, \omega) \geq 0, \quad \mathbf{v}, \omega \neq 0 .
$$

This requirement implies that $\mathbf{J}_{0}$ and $\mathbf{J}_{2}$ are positive tensors. Equation (7) can be written in the tensor-block matrix form as follows

$$
\widehat{K}_{\kappa}(\mathbf{v}, \boldsymbol{\omega})=\frac{1}{2}(\mathbf{v}, \boldsymbol{\omega}) \cdot \mathbb{J} \cdot\left(\begin{array}{c}
\mathbf{v} \\
\boldsymbol{\omega}
\end{array}\right), \quad \mathbb{J}=\left(\begin{array}{cc}
\mathbf{J}_{0} & \mathbf{J}_{1} \\
\mathbf{J}_{1}^{T} & \mathbf{J}_{2}
\end{array}\right) .
$$

The tensor-block matrix $\mathbb{J}$ corresponds to 6 by 6 matrix which is obviously symmetric and can be transformed into canonical (diagonal) form as follows

$$
\mathbb{J}=\left(\begin{array}{cccccc}
J_{1}^{(0)} & 0 & 0 & 0 & 0 & 0 \\
0 & J_{2}^{(0)} & 0 & 0 & 0 & 0 \\
0 & 0 & J_{3}^{(0)} & 0 & 0 & 0 \\
0 & 0 & 0 & J_{1}^{(2)} & 0 & 0 \\
0 & 0 & 0 & 0 & J_{2}^{(2)} & 0 \\
0 & 0 & 0 & 0 & 0 & J_{3}^{(2)}
\end{array}\right) .
$$

Here $J_{k}^{(0)}$ and $J_{k}^{(2)}, k=1,2,3$, can be treated as a diagonal elements of tensors $\mathbf{J}_{0}$ and $\mathbf{J}_{2}$, respectively, whereas $\mathbf{J}_{1}=\mathbf{0}$. On the other hand one has to be aware of such straightforward interpretations, as here 
we deal with the three-dimensional space and $\mathbf{J}_{0}$ and $\mathbf{J}_{2}$ are tensors given in this space. In this case the simultaneous diagonalization of both $\mathbf{J}_{0}$ and $\mathbf{J}_{2}$ is possible if the latter have the same eigen-vectors.

Another interpretation of (7) can be obtained if we consider a material particle $x$ of a micropolar medium as a rigid body of small size. In this case $\mathbf{J}_{0}$ depends on the mass of this particle and takes the form $\mathbf{J}_{0}=m \mathbf{1}$, where $m$ is a mass of $x$, whereas $\mathbf{J}_{2}=\mathbf{J}_{2}^{T}$ depends on mass and shape of $x$, see, e.g., [29]. In particular, in principal axes of inertia $\mathbf{J}_{2}$ takes a diagonal form. With this interpretation we can always consider both tensors of inertia as diagonal ones only in principal axes of inertia, in general. As the tensor of inertia of a rigid body depends also on the choice of an origin of a coordinate frame, $\mathbf{J}_{1} \neq \mathbf{0}$ corresponds to the case when the origin does not coincide with the center of mass of $x$, see, e.g., formula (4.7.7) for the kinetic energy in ([29], p. 171). So $\mathbf{J}_{1}$ can be called a relative microinertia tensor.

As an example, one can consider the simplest form of $\widehat{K}$

$$
\widehat{K}_{\kappa}(\mathbf{v}, \omega)=\frac{1}{2} \mathbf{v} \cdot \mathbf{v}+\frac{1}{2} \gamma \omega \cdot \omega,
$$

where $\gamma$ is a scalar measure of rotational inertia [21]. Equation (10) can be associated with material particles of spherical shape. In the tensor-block matrix form, $\mathbb{J}$ reads as follows

$$
\mathbb{J}=\left(\begin{array}{llllll}
1 & 0 & 0 & 0 & 0 & 0 \\
0 & 1 & 0 & 0 & 0 & 0 \\
0 & 0 & 1 & 0 & 0 & 0 \\
0 & 0 & 0 & \gamma & 0 & 0 \\
0 & 0 & 0 & 0 & \gamma & 0 \\
0 & 0 & 0 & 0 & 0 & \gamma
\end{array}\right) .
$$

However, in the following we intend to consider general case of the kinetic energy that is without any assumptions on the coordinate frame and corresponding restrictions for the microinertia tensors. Moreover, as we intend to analyze the dependence on the choice of a reference placement such general framework seems to be preferable.

Using an analogy with rigid body dynamics we introduce the referential microinertia tensors, so we get

$$
\mathbf{J}_{0}^{\kappa}=\mathbf{H}^{T} \cdot \mathbf{J}_{0} \cdot \mathbf{H}, \quad \mathbf{J}_{1}^{\kappa}=(\operatorname{det} \mathbf{H}) \mathbf{H}^{T} \cdot \mathbf{J}_{1} \cdot \mathbf{H}, \quad \mathbf{J}_{2}^{\kappa}=\mathbf{H}^{T} \cdot \mathbf{J}_{2} \cdot \mathbf{H} .
$$

Here we take into account that unlike $\mathbf{v}$, the angular velocity $\omega$ is an pseudovector, see, e.g., [33]. So $\mathbf{J}_{1}^{\kappa}$ is a second-order pseudotensor. In this case if we assume that $\mathbf{J}_{i}^{\kappa}, i=0,1,2$, are constant, and the stresses and couple stresses vanish in a micropolar medium, the equations of motion take exactly the form of equations of motion of a rigid body [29].

Following [23] with the principle of equipresence we assume that $\mathbf{J}_{0}, \mathbf{J}_{1}$ and $\mathbf{J}_{2}$ are functions of $\mathbf{x}$, $\mathbf{y}, \mathbf{F} \equiv \operatorname{Grad} \mathbf{y}, \mathbf{H}, \operatorname{Grad} \mathbf{H}, \mathbf{B}$, and $t$, in general,

$$
\mathbf{J}_{i}=\mathbf{J}_{i}(\mathbf{y}, \mathbf{H}, \mathbf{F}, \operatorname{Grad} \mathbf{H} ; \mathbf{x}, \mathbf{B}), \quad i=0,1,2 .
$$

In fact, considering as an example consolidation phenomena in granular media or suspensions, one can expect rather complex dependence of the microinertia tensors. Let us also note that there is no further requirements on $K_{\kappa}$ following from the material frame-indifference principle. It results in a certain difference in a possible representation of $K_{\kappa}$ in comparison with $W_{\kappa}$. Indeed, we see that $W_{\mathcal{K}}$ does not depend on $\mathbf{H}$ itself whereas for $K_{\mathcal{K}}$ such dependence is natural, see (7) with microinertia tensors given by (11).

In what follows we consider the following form of the microinertia tensors

$$
\begin{gathered}
\mathbf{J}_{0}=\mathbf{H} \cdot \mathbf{J}_{0}^{\kappa} \cdot \mathbf{H}^{T}, \quad \mathbf{J}_{1}=(\operatorname{det} \mathbf{H}) \mathbf{H} \cdot \mathbf{J}_{1}^{\kappa} \cdot \mathbf{H}^{T}, \quad \mathbf{J}_{2}=\mathbf{H} \cdot \mathbf{J}_{2}^{\kappa} \cdot \mathbf{H}^{T}, \\
\mathbf{J}_{i}^{\kappa}=\mathbf{J}_{i}^{\kappa}(\mathbf{U}, \mathbf{V} ; \mathbf{x}, \mathbf{B}), \quad i=0,1,2 .
\end{gathered}
$$


Here for the referential microinertia tensors we consider their dependence on the Lagrangian strains, the position vector $\mathbf{x}$ and the referential microcurvature tensor $\mathbf{B}$. The dependence on $\mathbf{x}$ and $\mathbf{B}$ is necessary for modelling of dynamically inhomogeneous materials.

\section{Local Material Symmetry Group}

Definition of the material symmetry group given in $[27,28]$ was based on invariance of $W_{\kappa}$ under replacements of the reference placement. Here we intend to extend it considering invariance properties of $K_{\kappa}$. Let us introduce another reference placement $\kappa_{*}=\kappa_{*}(\mathcal{B})$. In $\kappa_{*}$ the position of a material particle $x \in \mathcal{B}$ is given by the position vector $\mathbf{x}_{*}$ whereas its orientation is fixed by orthonormal directors $\mathbf{g}_{* k}$. Let $\mathbf{P}=\operatorname{Grad} \mathbf{x}_{*}, \operatorname{det} \mathbf{P} \neq 0$, be the deformation gradient tensor transforming $\kappa$ into $\kappa_{*}$, and $\mathbf{R}$ be the orthogonal tensor defined as $\mathbf{R}=\mathbf{g}_{* k} \otimes \mathbf{g}_{k}$. Here we consider static mappings $\kappa \rightarrow \kappa_{*}$. So we get

$$
d \mathbf{x}_{*}=\mathbf{P} \cdot d \mathbf{x}, \quad \mathbf{g}_{* k}=\mathbf{R} \cdot \mathbf{g}_{k}, \quad k=1,2,3 .
$$

In what follows we denote all fields associated with $\kappa_{*}$ by the lower index $*$. We have the following transformation rules $[27,28]$

$$
\mathbf{F}=\mathbf{F}_{*} \cdot \mathbf{P}, \quad \mathbf{H}=\mathbf{H}_{*} \cdot \mathbf{R},
$$

where $\mathbf{F}_{*}$ and $\mathbf{H}_{*}$ are the deformation gradient and the microrotation tensor calculated in $\kappa_{*}$, respectively. In addition we get the transformations of strain measures and the microcurvature tensor given by

$$
\mathbf{U}_{*}=\mathbf{R} \cdot(\mathbf{U}+\mathbf{1}) \cdot \mathbf{P}^{-1}-\mathbf{1}, \quad \mathbf{V}_{*}=(\operatorname{det} \mathbf{R}) \mathbf{R} \cdot \mathbf{V} \cdot \mathbf{P}^{-1}+\mathbf{L}, \quad \mathbf{B}_{*}=(\operatorname{det} \mathbf{R}) \mathbf{R} \cdot \mathbf{B} \cdot \mathbf{P}^{-1}-\mathbf{L},
$$

where

$$
\mathbf{L}=\mathbf{R} \cdot \mathbf{W} \cdot \mathbf{P}^{-1}, \quad \mathbf{W}=\frac{1}{2}(\mathbf{1} \times \mathbf{1}):\left(\mathbf{R} \cdot \operatorname{Grad} \mathbf{R}^{T}\right) .
$$

The constitutive equations naturally depend upon the choice of the reference placement, in general. Here we consider a particular class of possible choices and corresponding reference placements which leave unchanged the form of the constitutive equations. In other words, we are looking for a class of reference placements and related relations between reference placements in this class, for which kinetic and strain energy have the same form. Such transformations we call here invariant transformations. As in nonlinear elasticity, knowing all invariant transformations we can formulate the precise definitions of the elastic fluid as a material independent on any choice of a reference placements, the solid as a material which symmetry group consist orthogonal transformations, or the subfluid as an intermediate class of materials [26]. Furthermore, the group theory allows one to introduce notions of various anisotropic continua.

We introduce the elastic strain energy density $W_{*}$ and the kinetic energy density $K_{*}$ relative to new reference placement $\kappa_{*}$. In general, these dependencies may be different than that of $W_{\kappa}$ and $K_{\kappa}$. However, the stored energy in any part $\mathcal{P}$ of $\mathcal{B}$ of an elastic medium should be conserved, so that

$$
\int_{V_{\kappa}} W_{\kappa} d V_{\kappa}=\int_{V_{*}} W_{*} d V_{*}
$$

where $V_{\kappa}=\kappa(\mathcal{P})$ and $V_{*}=\kappa_{*}(\mathcal{P})$ are the volumes of $P$ in $\kappa$ and $\kappa_{*}$, respectively. Indeed, functions $W_{\kappa}$ and $W_{*}$ describe the strain energy density of the same deformed state of $\mathcal{P}$, while integrals in (17) present the same strain energy stored in $\mathcal{P}$. So from (17) it follows that $W_{*}$ and $W_{\mathcal{K}}$ are related by

$$
|\operatorname{det} \mathbf{P}| W_{*}\left(\mathbf{U}_{*}, \mathbf{V}_{*} ; \mathbf{x}, \mathbf{B}_{*}\right)=W_{\kappa}(\mathbf{U}, \mathbf{V} ; \mathbf{x}, \mathbf{B}),
$$


where $\mathbf{U}_{*}, \mathbf{V}_{*}$, and $\mathbf{B}_{*}$ are given by (15). From physical point of view any invariant transformation of the reference placement should preserve the elementary volume and referential mass density. So we have $\rho_{*}=\rho_{\kappa}$. As we have the mass balance $|\operatorname{det} \mathbf{P}| \rho_{*}=\rho_{\kappa}$, the tensor $\mathbf{P}$ should be an unimodular tensor, for which $|\operatorname{det} \mathbf{P}|=1$.

Similarly, the kinetic energy of any part $\mathcal{P}$ of $\mathcal{B}$ should be also conserved. So we get that

$$
\int_{V_{\kappa}} K_{\kappa} d V_{\kappa}=\int_{V_{*}} K_{*} d V_{*}
$$

for any part $\mathcal{P}$ of $\mathcal{B}$. As a result, we have

$$
|\operatorname{det} \mathbf{P}| K_{*}=K_{\kappa} .
$$

As $\mathbf{v}$ and $\omega$ are independent of the choice of the reference placement, the kinetic energy density depends on the reference placement through the mass density $\rho_{\kappa}$ and the microinertia tensors $\mathbf{J}_{i}$. As $|\operatorname{det} \mathbf{P}|=1$ the kinetic energy density depends on the reference placement only through $\mathbf{J}_{i}$. Using (12) we get the relation between $\mathbf{J}_{i}^{\kappa}$ and $\mathbf{J}_{i}^{*}$

$$
\mathbf{J}_{i}^{*}\left(\mathbf{U}_{*}, \mathbf{V}_{*} ; \mathbf{x}, \mathbf{B}_{*}\right)=(\operatorname{det} \mathbf{R})^{i} \mathbf{R} \cdot \mathbf{J}_{i}^{\kappa}(\mathbf{U}, \mathbf{V} ; \mathbf{x}, \mathbf{B}) \cdot \mathbf{R}^{T}, \quad i=0,1,2 .
$$

Using these transformation properties of the strain energy density and the microinertia tensors we can formulate the definition of the local material symmetry group. To this end we use the following nomenclature:

Orth $=\left\{\mathbf{Q}: \mathbf{Q}^{-1}=\mathbf{Q}^{T}, \operatorname{det} \mathbf{Q}= \pm 1\right\}$ - the group of orthogonal tensors with regard to multiplication;

Unim $=\{\mathbf{P}: \operatorname{det} \mathbf{P}= \pm 1\}-$ the unimodular group with regard to multiplication;

Lin $=\{\mathbf{L}\}-$ the linear group with regard to addition.

Generalizing $[27,28]$ we formulate new

Definition 1. By the local material symmetry group $\mathcal{G}_{\kappa}$ at $x$ of the micropolar hyperelastic continuum we call all sets of ordered triples of tensors

$$
\mathbb{X}=(\mathbf{P} \in \text { Unim, } \mathbf{R} \in \text { Orth, } \mathbf{L} \in \text { Lin }),
$$

satisfying the relations

$$
\begin{aligned}
& W_{\kappa}(\mathbf{U}, \mathbf{V} ; \mathbf{B})=W_{\kappa}\left[\mathbf{R} \cdot \mathbf{U} \cdot \mathbf{P}^{-1}+\mathbf{R} \cdot \mathbf{P}^{-1}-\mathbf{1},(\operatorname{det} \mathbf{R}) \mathbf{R} \cdot \mathbf{V} \cdot \mathbf{P}^{-1}+\mathbf{L} ;(\operatorname{det} \mathbf{R}) \mathbf{R} \cdot \mathbf{B} \cdot \mathbf{P}^{-1}-\mathbf{L}\right], \\
& \mathbf{J}_{i}^{\kappa}\left[\mathbf{R} \cdot \mathbf{U} \cdot \mathbf{P}^{-1}+\mathbf{R} \cdot \mathbf{P}^{-1}-\mathbf{1},(\operatorname{det} \mathbf{R}) \mathbf{R} \cdot \mathbf{V} \cdot \mathbf{P}^{-1}+\mathbf{L} ;(\operatorname{det} \mathbf{R}) \mathbf{R} \cdot \mathbf{B} \cdot \mathbf{P}^{-1}-\mathbf{L}\right] \\
& =(\operatorname{det} \mathbf{R})^{i} \mathbf{R} \cdot \mathbf{J}_{i}^{\kappa}(\mathbf{U}, \mathbf{V} ; \mathbf{x}, \mathbf{B}) \cdot \mathbf{R}^{T}, \quad i=0,1,2
\end{aligned}
$$

for any tensors $\mathbf{U}, \mathbf{V}, \mathbf{B}$ in domain of definition of the functions $W_{\kappa}$ and $\mathbf{J}_{i}^{\kappa}$.

The set $\mathcal{G}_{\kappa}$ is the group relative to the group operation o defined by

$$
\left(\mathbf{P}_{1}, \mathbf{R}_{1}, \mathbf{L}_{1}\right) \circ\left(\mathbf{P}_{2}, \mathbf{R}_{2}, \mathbf{L}_{2}\right)=\left[\mathbf{P}_{1} \cdot \mathbf{P}_{2}, \mathbf{R}_{1} \cdot \mathbf{R}_{2}, \mathbf{L}_{1}+\left(\operatorname{det} \mathbf{R}_{1}\right) \mathbf{R}_{1} \cdot \mathbf{L}_{2} \cdot \mathbf{P}_{1}^{-1}\right] .
$$

In Definition 1 we take into account that $\mathbf{U}, \mathbf{J}_{0}^{\kappa}$ and $\mathbf{J}_{2}^{\kappa}$ are polar (true) tensors, whereas $\mathbf{V}, \mathbf{B}$, $\mathbf{J}_{1}^{\kappa}$ are axial (pseudo)tensors. The difference between polar tensors and pseudotensors appears when one consider mirror reflection transformations, which change right-handed frame into left-handed and vice versa, see the discussion in [33] where other examples of pseudoscalars, pseudovectors and pseudotensors are given. 
As an example, let us assume that $\mathbf{J}_{i}^{\kappa}$ are constant tensors. Then the proposed definition states that the symmetry group includes symmetries of the strain energy density and the symmetries of microinertia tensors. So the material symmetry depends on the both symmetries. Let us consider as an example the modified definition of an isotropic micropolar solid following from Definition 1. As in case of nonlinear elasticity the symmetry group consists of orthogonal tensors only.

Definition 2 (Isotropic material). The micropolar medium is called isotropic solid at $x$ if there exists a reference placement $\kappa$, called undistorted, such that the material symmetry group relative to $\kappa$ takes the form

$$
\mathcal{G}_{\kappa}=\mathcal{S}_{\kappa} \equiv\{(\mathbf{P}=\mathbf{Q}, \mathbf{Q}, \mathbf{0}): \quad \mathbf{Q} \in \text { Orth }\}
$$

Note that in (20) $\mathbf{Q}$ denotes arbitrary orthogonal tensor whereas $\mathbf{0}$ means the second-order zero tensor. So the symmetry group is based on elements of Orth.

This definition means that the strain energy density and the microinertia tensors are isotropic functions of their arguments:

$$
\begin{aligned}
& W_{\kappa}(\mathbf{U}, \mathbf{V} ; \mathbf{B})=W_{\kappa}\left[\mathbf{Q} \cdot \mathbf{U} \cdot \mathbf{Q}^{T},(\operatorname{det} \mathbf{Q}) \mathbf{Q} \cdot \mathbf{V} \cdot \mathbf{Q}^{T} ;(\operatorname{det} \mathbf{Q}) \mathbf{Q} \cdot \mathbf{B} \cdot \mathbf{Q}^{T}\right], \\
& \mathbf{J}_{i}^{\kappa}\left[\mathbf{Q} \cdot \mathbf{U} \cdot \mathbf{Q}^{T},(\operatorname{det} \mathbf{Q}) \mathbf{Q} \cdot \mathbf{V} \cdot \mathbf{Q}^{T} ;(\operatorname{det} \mathbf{Q}) \mathbf{Q} \cdot \mathbf{B} \cdot \mathbf{Q}^{T}\right]=(\operatorname{det} \mathbf{Q})^{i} \mathbf{Q} \cdot \mathbf{J}_{i}^{\kappa}(\mathbf{U}, \mathbf{V} ; \mathbf{x}, \mathbf{B}) \cdot \mathbf{Q}^{T}, \quad i=0,1,2
\end{aligned}
$$

So one can apply the representations of isotropic functions as in $[27,28,33]$.

In particular, if $\mathbf{J}_{i}^{\kappa}$ do not depend on $\mathbf{U}, \mathbf{V}, \mathbf{B}$ as in rigid body dynamics, the last properties means that $\mathbf{J}_{0}^{\kappa}, \mathbf{J}_{1}^{\kappa}$ and $\mathbf{J}_{2}^{\kappa}$ are isotropic tensors. Any isotropic second-order tensor has the form $\lambda \mathbf{1}$, where $\lambda$ is a scalar, whereas a second-order pseudotensor should be zero, see, e.g., [33]. So in this case we can assume that $\mathbf{J}_{0}^{\kappa}=\mathbf{1}, \mathbf{J}_{2}^{\kappa}=\gamma \mathbf{1}$ and the kinetic energy density takes the form (10). In other words, the kinetic energy density (10) can be associated with an isotropic micropolar solid.

\section{Conclusions}

Here we have extended the definition of the material symmetry group $[27,28]$ considering invariance properties of the kinetic energy density. Invariant properties of the kinetic energy density express through symmetries of the three microinertia tensors. From the physical point of view, in new definition we unify static and dynamic responses which should be distinguished in general. For example, in statics a medium can demonstrate an isotropic response whereas its anisotropic properties appear in dynamics. This situation can be observed for example if the strain energy density is an isotropic scalar function whereas the microinertia tensors are not isotropic. In forthcoming papers we consider some applications of the considered approach to dynamic problems for micropolar solids, fluids, and subfluids.

Discussed here approach can be also applied to other generalized media with complex form of kinetic constitutive equations. Among such media it is worth to mention the Toupin-Mindlin strain gradient elasticity [24,33,34]. In these media we face to higher order elastic moduli tensors, see [35-37] where the analysis of their structure was performed. Within this model kinetic energy may depend on velocity and its spatial gradients, that extends a possible forms of the kinetic energy density. The definitions of the symmetry group for strain gradient media were given in [38-40]. Another medium with non-trivial form of kinetic energy is a micromorphic continuum $[21,24,33]$ for which the material symmetry group was discussed in [41].

It is worth also to mention here symmetry analysis for thin-walled structures, i.e., membranes, plates and shells. In fact, the rotational inertia was known from landscape works by Timoshenko [42] and Mindlin [43]. In [44,45] the symmetry group analysis was also performed on the base of static analysis.

So the analysis of the material symmetry groups and corresponding constitutive relations could be also extended considering invariance properties of kinetic constitutive relations for these media. 
Author Contributions: Conceptualization, V.A.E.; methodology, V.A.E.; validation, V.A.E. and V.K.-Z.; formal analysis, V.A.E.; investigation, V.A.E. and V.K.-Z.; writing-original draft preparation, V.A.E. and V.K.-Z.; writing-review and editing, V.A.E. and V.K.-Z.; project administration, V.A.E.; funding acquisition, V.A.E. All authors have read and agreed to the published version of the manuscript.

Funding: This research was funded by Government of the Russian Federation (MEGAGrant program) grant number 14.Y26.31.0031. The APC was funded by MDPI.

Conflicts of Interest: The authors declare no conflict of interest. The funders had no role in the design of the study; in the collection, analyses, or interpretation of data; in the writing of the manuscript, or in the decision to publish the results.

\section{References}

1. Cosserat, E.; Cosserat, F. Théorie des corps déformables; Herman et Fils: Paris, France, 1909.

2. Tejchman, J. Shear Localization in Granular Bodies with Micro-Polar Hypoplasticity; Springer: Berlin, Germany, 2008.

3. Vardoulakis, I. Cosserat Continuum Mechanics: With Applications to Granular Media; Springer: Cham, Switzerland, 2019.

4. Rueger, Z.; Lakes, R.S. Experimental Cosserat elasticity in open-cell polymer foam. Philos. Mag. 2016, 96, 93-111. [CrossRef]

5. Rueger, Z.; Lakes, R. Experimental study of elastic constants of a dense foam with weak Cosserat coupling. J. Elast. 2019, 137, 101-115. [CrossRef]

6. Reccia, E.; De Bellis, M.L.; Trovalusci, P.; Masiani, R. Sensitivity to material contrast in homogenization of random particle composites as micropolar continua. Compos. Part B Eng. 2018, 136, 39-45. [CrossRef]

7. Hütter, G. On the micro-macro relation for the microdeformation in the homogenization towards micromorphic and micropolar continua. J. Mech. Phys. Solids 2019, 127, 62-79. [CrossRef]

8. Besdo, D. Towards a Cosserat-theory describing motion of an originally rectangular structure of blocks. Arch. Appl. Mech. 2010, 80, 25-45. [CrossRef]

9. Leonetti, L.; Greco, F.; Trovalusci, P.; Luciano, R.; Masiani, R. A multiscale damage analysis of periodic composites using a couple-stress/Cauchy multidomain model: Application to masonry structures. Compos. Part B Eng. 2018, 141, 50-59. [CrossRef]

10. Park, H.C.; Lakes, R.S. Cosserat micromechanics of human bone: Strain redistribution by a hydration-sensitive constituent. J. Biomech. 1986, 19, 385-397. [CrossRef]

11. Goda, I.; Assidi, M.; Belouettar, S.; Ganghoffer, J.F. A micropolar anisotropic constitutive model of cancellous bone from discrete homogenization. J. Mech. Behav. Biomed. Mater. 2012, 16, 87-108. [CrossRef]

12. Suiker, A.S.J.; Metrikine, A.V.; de Borst, R. Comparison of wave propagation characteristics of the Cosserat continuum model and corresponding discrete lattice models. Int. J. Solids Struct. 2001, 38, 1563-1583. [CrossRef]

13. Abdoul-Anziz, H.; Seppecher, P. Strain gradient and generalized continua obtained by homogenizing frame lattices. Math. Mech. Complex Syst. 2018, 6, 213-250. [CrossRef]

14. Rueger, Z.; Ha, C.S.; Lakes, R.S. Cosserat elastic lattices. Meccanica 2019, 54, 1983-1999. [CrossRef]

15. Eremeyev, V.A. Two- and three-dimensional elastic networks with rigid junctions: Modelling within the theory of micropolar shells and solids. Acta Mech. 2019, 230, 3875-3887. [CrossRef]

16. Shirani, M.; Steigmann, D.J. A Cosserat model of elastic solids reinforced by a family of curved and twisted fibers. Symmetry 2020, 12, 1133. [CrossRef]

17. Eringen, A.C. Continuum theory of dense rigid suspensions. Rheol. Acta 1991, 30, 23-32. [CrossRef]

18. Eringen, A.C. A continuum theory of dense suspensions. ZAMP 2005, 56, 529-547. [CrossRef]

19. Altenbach, H.; Naumenko, K.; Zhilin, P.A. A micro-polar theory for binary media with application to phase-transitional flow of fiber suspensions. Contin. Mech. Thermodyn. 2003, 15, 539-570. [CrossRef]

20. Eringen, A.C.; Kafadar, C.B. Polar field theories. In Continuum Physics; Eringen, A.C., Ed.; Academic Press: New York, 1976; Volume IV, pp. 1-75.

21. Eringen, A.C. Microcontinuum Field Theory. I. Foundations and Solids; Springer: New York, NY, USA, 1999.

22. Eringen, A.C. Microcontinuum Field Theory. II. Fluent Media; Springer: New York, NY, USA, 2001.

23. Eremeyev, V.A.; Lebedev, L.P.; Altenbach, H. Foundations of Micropolar Mechanics; Springer-Briefs in Applied Sciences and Technologies; Springer: Heidelberg, Germany, 2013. 
24. Maugin, G.A. Non-Classical Continuum Mechanics: A Dictionary; Springer: Singapore, 2017.

25. Kafadar, C.B.; Eringen, A.C. Micropolar media-I. The classical theory. Int. J. Eng. Sci. 1971, 9, $271-305$. [CrossRef]

26. Truesdell, C.; Noll, W. The Non-Linear Field Theories of Mechanics, 3rd ed.; Springer: Berlin, Germany, 2004.

27. Eremeyev, V.A.; Pietraszkiewicz, W. Material symmetry group of the non-linear polar-elastic continuum. Int. J. Solids Struct. 2012, 49, 1993-2005. [CrossRef]

28. Eremeyev, V.A.; Pietraszkiewicz, W. Material symmetry group and constitutive equations of micropolar anisotropic elastic solids. Math. Mech. Solids 2016, 21, 210-221. [CrossRef]

29. Lurie, A.I. Analytical Mechanics; Springer: Berlin, Germany, 2001.

30. Müller, W.H.; Vilchevskaya, E.N. Micropolar theory with production of rotational inertia: A rational mechanics approach. In Generalized Models and Non-Classical Approaches in Complex Materials 1; Springer: Cham, Switzerland, 2018; pp. 581-606.

31. Pietraszkiewicz, W.; Eremeyev, V.A. On natural strain measures of the non-linear micropolar continuum. Int. J. Solids Struct. 2009, 46, 774-787. [CrossRef]

32. Pietraszkiewicz, W. Refined resultant thermomechanics of shells. Int. J. Eng. Sci. 2011, 49, 1112-1124. [CrossRef]

33. Eremeyev, V.A.; Cloud, M.J.; Lebedev, L.P. Applications of Tensor Analysis in Continuum Mechanics; World Scientific: Hackensack, NJ, USA, 2018.

34. Bertram, A.; Forest, S. (Eds.) Mechanics of Strain Gradient Materials; Springer International Publishing: Cham, Switzerland, 2020.

35. Auffray, N.; Le Quang, H.; He, Q.C. Matrix representations for 3D strain-gradient elasticity. J. Mech. Phys. Solids 2013, 61, 1202-1223. [CrossRef]

36. Auffray, N.; Dirrenberger, J.; Rosi, G. A complete description of bi-dimensional anisotropic strain-gradient elasticity. Int. J. Solids Struct. 2015, 69, 195-206. [CrossRef]

37. Dell'Isola, F.; Sciarra, G.; Vidoli, S. Generalized Hooke's law for isotropic second gradient materials. R. Soc. Lond. Proc. Ser. A 2009, 465, 2177-2196. [CrossRef]

38. Murdoch, A.I. Symmetry considerations for materials of second grade. J. Elast. 1979, 9, 43-50. [CrossRef]

39. Elzanowski, M.; Epstein, M. The symmetry group of second-grade materials. Int. J. Non-Linear Mech. 1992, 27, 635-638. [CrossRef]

40. Reiher, J.C.; Bertram, A. Finite third-order gradient elasticity and thermoelasticity. J. Elast. 2018, 133, $223-252$. [CrossRef]

41. Eremeyev, V.A. On the material symmetry group for micromorphic media with applications to granular materials. Mech. Res. Commun. 2018, 94, 8-12. [CrossRef]

42. Timoshenko, S.P. On the correnction for shear of the differential equation for transverse vibrations of prismatic bars. Philos. Mag. Ser. 6 1921, 41, 744-746. [CrossRef]

43. Mindlin, R.D. Influence of rotatory inertia and shear on flexural motions of isotropic elastic plates. Trans. Asme. J. Appl. Mech. 1951, 18, 31-38.

44. Murdoch, A.I.; Cohen, H. Symmetry considerations for material surfaces. Arch. Ration. Mech. Anal. 1979, 72, 61-98. [CrossRef]

45. Eremeyev, V.A.; Pietraszkiewicz, W. Local symmetry group in the general theory of elastic shells. J. Elast. 2006, 85, 125-152. [CrossRef]

(c) 2020 by the authors. Licensee MDPI, Basel, Switzerland. This article is an open access article distributed under the terms and conditions of the Creative Commons Attribution (CC BY) license (http://creativecommons.org/licenses/by/4.0/). 